\title{
Effect of Methadone and Tramadol Opioids on Stem Cells Based on Integrated Plasmonic-Ellipsometry Technique
}

\author{
Tannaz Asadishad $^{1}$, Foozieh Sohrabi ${ }^{\circledR}$, Maryam Hakimi' ${ }^{2}$, Mohammad Hossein Ghazimoradi², \\ Tayebeh Mahinroosta1 ${ }^{1}$ Seyedeh Mehri Hamidi ${ }^{1 * \mathbb{D}}$, Shirin Farivar ${ }^{2,3}{ }^{\mathbb{D}}$ \\ 'Magneto-plasmonic Lab, Laser and Plasma Research Institute, Shahid Beheshti University, Tehran, Iran \\ ${ }^{2}$ Life Science and Biotechnology Faculty, Shahid Beheshti University, Tehran, Iran \\ ${ }^{3}$ Laser Application in Medical Sciences Research Center, Shahid Beheshti University of Medical Sciences, Tehran, \\ Iran
}

\section{*Correspondence to Seyedeh Mehri Hamidi, Magneto-plasmonic Lab, Laser and Plasma Research Institute, Shahid Beheshti University, Tehran, Iran \\ Email: m_hamidi@sbu.ac.ir}

Received: November 6, 2020 Accepted: April 5, 2021 Published online August 26, 2021

\begin{abstract}
Introduction: Plasmonic biosensors provide high sensitivity in detecting the low amount of biomarkers and pharmaceutical drugs. We studied the mesenchyme cell activity under the treatment of common sedative drugs of methadone and tramadol using the integrated plasmonic-ellipsometry technique.

Methods: Mesenchymal stem cells were cultured on patterned plasmonic chips under the treatment of methadone and tramadol drugs. Three cultured chips were kept non-treated as the control ones. The plasmonic-ellipsometry technique was applied to study the signaling characteristic of the cells affected by these two drugs. In this technique, optical information regarding the amplitude ratio and phase change between $\mathrm{p}$ - and s-polarized light was recorded.

Results: This drug treatment could affect the spectral plasmonic resonance and subsequently the phase shift $(\Delta)$ and the amplitude ratio $(\Psi)$ values under $p$ - and s-polarized impinging light. A more significant $\Delta$ value for tramadol treatment meant that the phase split was larger between $p$ - and s-polarized light. Tramadol also had more prominent absolute $\Delta_{\text {eff }}$ and $\Psi_{\text {eff }}$ values in comparison with methadone.

Conclusion: We showed that tramadol caused more contrast in phase shift $(\Delta)$ and amplitude ratio $(\Psi)$ between $\mathrm{p}$ - and s-polarized impinging light for cultured stem cells in comparison with methadone. It means that tramadol differentiated more the optical responses for $\mathrm{p}$ - and s-polarized lights compared to methadone. Our proposed technique possesses the potential of quantitative and qualitative analysis of drugs on humans even on a cell scale.

Keywords: Plasmonic crystals; Ellipsometry; Methadone; Tramadol; Mesenchymal stem cells.
\end{abstract}

\section{Introduction}

There are numerous substitutional sedative drugs with various side effects on different organs like respiratory and neural systems. ${ }^{1,2}$ Among these, methadone and tramadol are the drugs that are frequently prescribed for killing the pain or releasing the patients from other addicting drugs. ${ }^{3,4}$ More specifically, these drugs influence different mental (e.g., pain, awareness, etc) ${ }^{5}$ and physical parts (e.g., skin, cardiac effect, etc). ${ }^{6,7}$ They sometimes play a role in reducing cancer pain as well. ${ }^{8}$ Methadone is the agonist of the $\mu$-opioid receptor, which can impact numerous physiological functions and plays a role in controlling pain perception and reward properties. ${ }^{9,10}$ Tramadol is a partial opioid agonist, which has more effects on $\mu, 5 \mathrm{TH}_{3}$ and $\alpha_{7}$ nicotine receptors compared to methadone. ${ }^{7,11}$ Prescribing an effective dosage of these drugs plays an important role since the dosage should be kept as low as possible due to adverse effect of opioids. In addition, their observation in biofluids (i.e., blood or urine) helps to detect drug abuse in addicts. One of the strong techniques for drug detection and its impacts on the cells is plasmonic sensing. Among optical techniques, the surface plasmon resonance (SPR) technique is an unlabeled, non-invasive, and actual-time approach that provides a chemical detection facility at low concentrations. ${ }^{12,13}$ The application of plasmonics in pharmaceutics allows SPR biosensors to detect molecular binding activities at low concentrations as well as cellular changes. ${ }^{14}$ El-Zahry ${ }^{15}$ has utilized localized surface plasmon resonance (LSPR) of Ag nanoparticles for parallel detection of the mixture of antihypertensive drugs of atenolol and amiloride pharmaceutical dosage forms as well as urine samples. Their method was based on the UV

Please cite this article as follows: Asadishad T, Sohrabi F, Hakimi M, Ghazimoradi MH, Mahinroosta T, Hamidi SM, Farivar S. Effect of methadone and tramadol opioids on stem cells based on integrated plasmonic-ellipsometry technique. J Lasers Med Sci. 2021;12:e46. doi:10.34172/jlms.2021.46 
absorption intensities of these mixed analytes. IzquierdoLorenzo et $\mathrm{al}^{16}$ have applied surface-enhanced Raman spectroscopy and LSPR to detect aminoglutethimide drug, which is an aromatase inhibitor and is used in illegal sport doping. Juhász et $\mathrm{a}^{17}$ have introduced a dynamic and thermodynamic assessment of a plasmonic biosensor for a fragmented receptor and a drug. Kampranis et $\mathrm{al}^{18}$ have investigated the interaction of coumarin and cyclothialidine drugs (MWt $<700 \mathrm{Da}$ ) with DNA gyrase, which was the target of some bacterial agents. They have demonstrated the residues on gyrase, which were essentials for drug binding. Su et $\mathrm{al}^{19}$ have fabricated plasmonic capsules of citrate-capped Au nanoparticles for analyzing the stimulant methamphetamine drug. Mozneb et $\mathrm{a}^{20}$ have used real-time SPR technology to characterize the contractility of cardiac cells in response to drug exposure of Blebbistatin and ATP. Jackman et $\mathrm{al}^{21}$ have developed a plasmonic nanohole sensor to trap viruslike particles and measure the virucide drugs. For this aim, the size effect of dengue virus particles was studied without and with the drug benefiting from nanoparticle tracking analysis and the spectral shift of the plasmonic resonances. Huang et $\mathrm{al}^{22}$ have synthesized SiO2@Ag nanoshells with tunable shell structures for diagnostics and therapeutics. They investigated the distribution of the drug in blood and the cerebrospinal fluid systems. They confirmed the role of blood-brain/cerebrospinal fluid-barriers for pharmacokinetics. Kong et $\mathrm{al}^{23}$ have suggested a plasmonic structure based on an enzymatic nanoreactor for monitoring the antidiabetic drug. The glucose was used by glucose oxidase to generate $\mathrm{H}_{2} \mathrm{O}_{2}$, which etched the $\mathrm{Ag}$ nanoparticles to circular shapes and caused the blueshift in the plasmonic absorption band. Their reported limit of detection was $5 \mathrm{nM}$ for acarbose. In this study, we fabricated 2D flexible plasmonic chips and studied the impact of methadone and tramadol drugs on cultured mesenchyme cells using the optical phase and amplitude sensitive plasmonic technique without any time-consuming labeling procedure. The Ellipsometry technique provides information on both the amplitude ratio and the phase delay between reflections of light beams with $\mathrm{p}$ and $\mathrm{s}$ polarizations. In comparison with reflections, this technique can detect changes with the enhanced signal-to-noise ratio. ${ }^{24}$ Although reflection fails to represent small biological changes, phase interrogation can reveal small changes in the cells caused by the drug treatment. In the Materials and Methods section, we discuss the simulation and fabrication of the sensing chip, cell culture on the chip surface and the optical measurement based on the plasmonic-ellipsometry technique for optical investigation of the effect of methadone and tramadol sedative drugs on stem cells. Subsequently, there are results and discussions on the optical detection of the changes in stem cells.

\section{Materials and Methods}

Fabrication of 2D Plasmonic Chip and Simulation

We proposed a $2 \mathrm{D}$ flexible plasmonic crystal as a costeffective sensing chip for mass production. Using the soft lithography technique, ${ }^{25}$ two-dimensional (2D) patterned poly-dimethyl siloxane (PDMS) substrates were fabricated. Charge-coupled devices (CCDs) were extracted from second-hand commercial cameras. These CCD molds were settled at the base of the petri dishes with double-sided tapes. The mixture of PDMS and its curing agent (10:1 aspect ratio) was poured on top of the molds and the sides were sealed using a glue gun to avoid leakage. The mold was put in the vacuum chamber for degassing following the heating step. In the heating step, the mold was inserted on the heater for $1 \mathrm{~h}$ (30 minutes at $50^{\circ}, 15$ minutes at $75^{\circ}$, and 15 minutes at $\left.100^{\circ}\right)$. After cooling for 24 hours, the patterned PDMS substrates with the inverse pattern of the CCD were peeled off from the mold using a sharp scalpel. $30 \mathrm{~nm}$ gold was coated on this layer using a sputtering machine. The fabrication process is illustrated in Figure 1. Twelve similar chips were fabricated using this process. Using scanning electron microscope (SEM) images, the fabricated structure had a periodicity of $2.16 \mu \mathrm{m}$ and a depth of $0.25 \mu \mathrm{m}$, as shown in Figure 1. We simulated the same structure with $30-\mathrm{nm}$ gold thickness using Lumerical FDTD solutions. The mesh size was $2 \mathrm{~nm}$ and $10 \mathrm{~nm}$ in the $\mathrm{Z}$-direction and the X-Y plane respectively. The unit cell and the simulation results are provided in Figure 1. Figure 1 shows the comparison of the ratio of reflections for $\mathrm{p}$ - and s-polarized impinging light $\left(\mathrm{R}_{\mathrm{p}} / \mathrm{R}_{\mathrm{s}}\right)$ in simulation and experiment. As seen, the main dips occurred at $483 \mathrm{~nm}$ and $547 \mathrm{~nm}$ for simulation results and at $455 \mathrm{~nm}$ and $526 \mathrm{~nm}$ for the experiment. There are $28 \mathrm{~nm}$ and $21 \mathrm{~nm}$ spectral differences for first and second dips, showing an acceptable match between simulation and experiment results.

\section{Cell Culture}

Mesenchymal stem cells were extracted from the femur bone marrow of the mouse. We used a scarified mouse from another study as a donation and only one bone from one mouse was used. For the cell culture of mesenchymal stem cells, the protocol from Soleimani and Nadri. ${ }^{26}$ was followed. The bone was cut and sterilized, and then the cells were extracted by syringing bone marrow by using $2 \mathrm{~mL}$ cell culture media; then they were cultured in the same medium. The medium consisted of RPMI1640, containing 1\% antibiotic and 20\% fetal bovine serum (FBS). The medium was changed every day with the fresh medium consisting of RPMI1640 (10\% FBS, and $1 \%$ pen strep) until the cells achieved $80 \%$ confluency. After that, the cells were trypsinized using trypsin $0.25 \%$ and centrifuged with $1000 \mathrm{rpm}$; then the supernatant was discarded and the pellet was re-suspended in a fresh medium. The chips were first sterilized with UV and 75\% ethanol before seeding cells. The confluent cells were again trypsinized as it was described. They were transferred to the chips in a 24-well plate, 

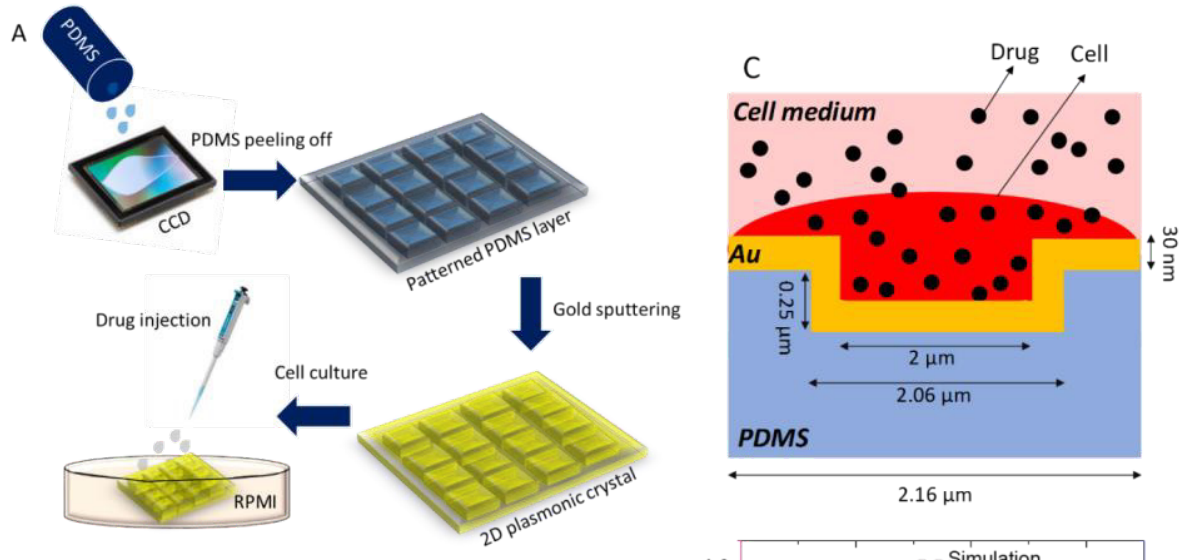

B
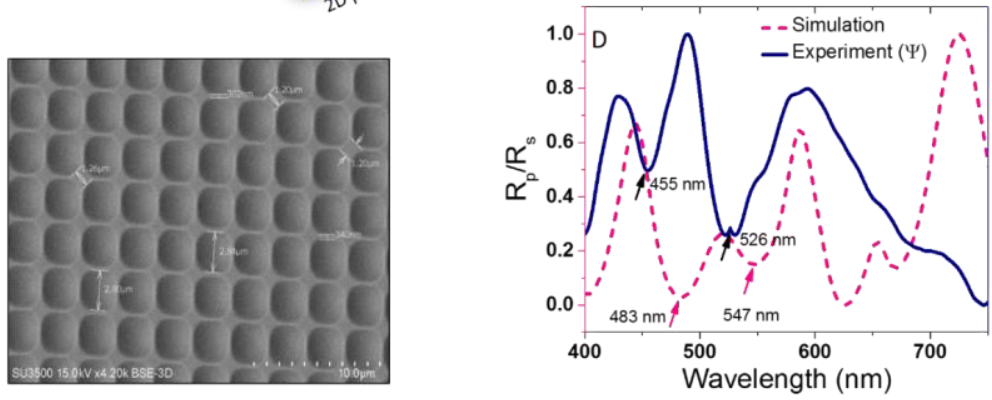

Figure 1. (A) schematic of the fabrication process for 2D plasmonic chips. The PDMS mixture was poured on the CCD mold, degassed and cured. The patterned PDMS was peeled off from its mold after $24 \mathrm{~h}$ following $30 \mathrm{~nm}$ gold sputtering. We carried out the cell culture and drug treatment on these chips. (B) SEM image of the fabricated structure. The fabricated structure on $1.4 \mathrm{~mm}$ PDMS had an average periodicity of $2.16 \mu \mathrm{m}$ with hollow semi-cubic patterns. These cubes were apart from each other at the average distance of $1.68 \mu \mathrm{m}$ (C) Schematic of the simulated unit cell. (D) Simulation results using Lumerical FDTD Solutions. The main dips occur at $483 \mathrm{~nm}$ and $547 \mathrm{~nm}$ for simulation results and at $455 \mathrm{~nm}$ and $526 \mathrm{~nm}$ for experimental results showing an acceptable match between simulation and experimental results.

and then the extra medium was added. In our previous studies on mesenchymal stem cells, we performed flow cytometry for stem cell markers with the same protocol as mentioned in Moghadam et $\mathrm{al}^{27}$ and the functional differentiation test in the mesenchymal stem cells was performed in Farivar et al. ${ }^{28}$ Drug treatment was performed five minutes before the optical measurement. (For the drug treatment procedure, the readers are referred to Brawanski et $\mathrm{al}^{2}$ and Plantz \& Hsu. ${ }^{29}$ Each of the pills was weighed separately, crushed and powdered in a mortar, dissolved in distilled water, and filtered to prepare $2 \mu \mathrm{M}$ tramadol and $2.17 \mu \mathrm{M}$ methadone solutions. From 12 chips, we had three uncultured chips for air measurement, three cultured chips with methadone treatment, three cultured chips with tramadol treatment, and three with no treatment. The real images of the cultured chips are shown in Figure 2. In this study, the chips were weakly fixed at the bottom of the plates and the cell attachment was satisfactory, similar to Saeidifard et $\mathrm{al}^{30}$ so we did not use poly-L-lysine that was used in our previous study. ${ }^{31}$

\section{Optical Setup}

For obtaining the reflection of the samples for s- and p-polarized impinging light, an open-optic setup was mounted. This setup consisted of broadband halogen fiber optic illuminator, collimator, lens, Glan-Taylor calcite polarizer (GT10-A), aperture, sample chamber, rotation stage, and
Ocean Optics spectrometer. By passing the Glan-Taylor calcite polarizer, the incident light obtained s- and p-polarizations. These components are shown schematically in Figure 2. The chips were mounted in the opening of the cubic chamber in a way that the top surface of the chips encountered the inside of the chamber, and the reflected light could be gathered from the backside of the chips. There was a small hole on the top surface of the chamber from which the fresh medium could be injected. The stage was rotated to provide an incident angle of $35^{\circ}$. The reflectance of the back of the sample at s- and p-polarization depended highly on the adsorption of biomolecules on the surface. ${ }^{32}$ Ellipsometry spectroscopy is a non-invasive method for SPR analysis with high resolution that gives knowledge on the ratio of the amplitude $(\Psi)$ and the phase shift $(\Delta)$ between the reflections of $s$ - and p-polarized impinging light. ${ }^{33,34}$ The complex reflection coefficient is $\rho=\frac{r_{p}}{r_{s}} \tan \Psi e^{i \Delta}$ where $\left|r_{p, s}\right|=\sqrt{R_{p, s}}$, and

$\Psi=\tan ^{(-1)} \sqrt{R_{p} / R_{s}}$,

$\Delta=\theta_{p}(\omega)-\theta_{s}(\omega)=-\frac{2 \pi}{\omega} P \int_{0}^{\infty}\left[\frac{\operatorname{Ln}\left[\sqrt{\frac{R_{p}\left(\omega^{\prime}\right)}{R_{s}\left(\omega^{\prime}\right)}}\right]}{\omega^{\prime 2}-\omega^{2}}\right.$.

$\mathrm{r}_{\mathrm{p}}$ and $\mathrm{r}_{\mathrm{s}}$ are the reflections for $\mathrm{p}$ - and s-polarized impinging light. The relations between ellipsometric parameters $(\Delta$, 


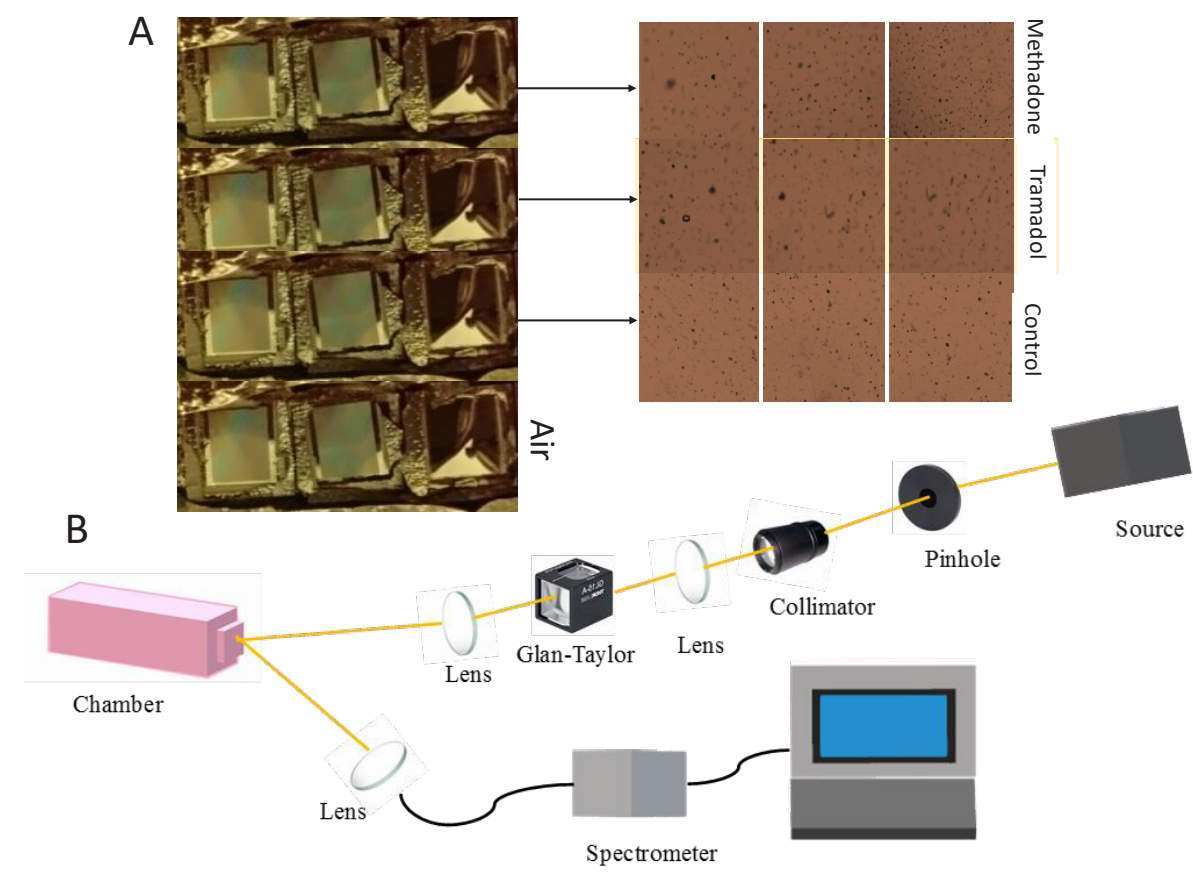

Figure 2. (A) Twelve fabricated chips. First and second rows were cultured with mesenchyme stem cells and treated with methadone and tramadol drugs respectively. The third row was cultured with no treatment. The last row was kept non-cultured for air measurements. (B) Optical setup for recording the reflections under s- and p-polarized incident light. The components were broadband halogen fiber optic illuminator, collimator, lens, Glan-Taylor calcite polarizer (GT10-A), aperture, sample chamber, rotation stage and Ocean spectrometer. Glan-Taylor calcite polarizer provided s- and p- polarizations. The chips were mounted in the opening of the cubic chamber in a way that the top surface of the chips faced the inner part of the chamber, and the reflected light could be gathered from the backside of the chips.

$\Psi)$ and their extraction technique are discussed in detail in Saeidifard et al. ${ }^{30}$

\section{Results and Discussion}

The reflections of all the samples for the incident light with $\mathrm{s}$ and $\mathrm{p}$ polarizations were recorded at the incident angle of $35^{\circ}$, and the ellipsometry parameters of $\Psi$ and $\Delta$ were extracted. As explained in Sohrabi and Hamidi, ${ }^{35}$ the plasmonic resonance emerges as a dip in the $\Psi$ parameter and as a peak in the $\Delta$ parameter. Due to the high signalto-noise ratio, the integrated plasmonic-ellipsometry technique has the capability to demonstrate minor resonances, which may not be observable in reflection spectra. ${ }^{36}$ Figure 3 shows the mean values of reflections and the corresponding $\Delta$ and $\Psi$ parameters. As seen, the plasmonic resonance dip is not clear in reflection. However, this resonance is conspicuous in $\Psi$ and $\Delta$ parameters. The resonance wavelength emerges at $550 \mathrm{~nm}$ for methadone and $475 \mathrm{~nm}$ for tramadol. The plasmonic resonance is redshifted for the methadone case and blueshifted for the tramadol case in comparison with the control sample. Multiple resonances in the control sample were originated from the diffraction property of the fabricated structure. Metallic periodic plasmonic crystal supports LSPR, where charge density oscillation is confined. These periodic arrays scatter light and produce diffractive waves with constructive and destructive patterns. The shape of nanoparticles/holes and the refractive index of the adjacent dielectric play a significant role in the excitation of the surface lattice resonances (SLRs). If $a$ is the polarizability of the single particle $(\alpha=\operatorname{Re}(\alpha)+\operatorname{Im}$ $(\alpha)$ ) and $S$ is the array factor, the resonance wavelength of the SLR locates at the crossing of $\operatorname{Re}(1 / \alpha)$ and $\operatorname{Re}(S)$ that is a function of the distance between the particles. ${ }^{37,38}$ The contrast between the $\operatorname{Im}(1 / \alpha)$ and $\operatorname{Im}(S)$ plays a role in the robustness and the width of SLR. In this structure, the interference between two modes occurs at various wavelengths proportional to the lattice constant. In our proposed $2 \mathrm{D}$ grating structure, the diffractive patterns and LSPRs can interfere to produce SLR interference patterns. Drug treatment directly affected the refractive index of the cells and subsequently the SLR modes.

As shown in Figure 4, for the $\Delta$ parameter, resonance occurs at [552 nm, $\left.3.74^{\circ}\right],\left[608 \mathrm{~nm}, 3.88^{\circ}\right]$, and [497 $\left.\mathrm{nm}, 5.58^{\circ}\right]$ for control, methadone and tramadol treated cases respectively. For the $\Psi$ parameter, resonance occurs at [529 nm, 43.7], [ $571 \mathrm{~nm}, 46.13]$, and [ $480 \mathrm{~nm}, 30.92]$ for control, methadone and tramadol treated cases respectively. Compared to the non-treated (i.e. control) case, the spectral position is redshifted for methadone and blueshifted for tramadol cases. This redshift meant that the methadone treatment raised the refractive index of the biosample and tramadol reduced the refractive index of the biosample. The largest $\Delta$ value was for the tramadol 

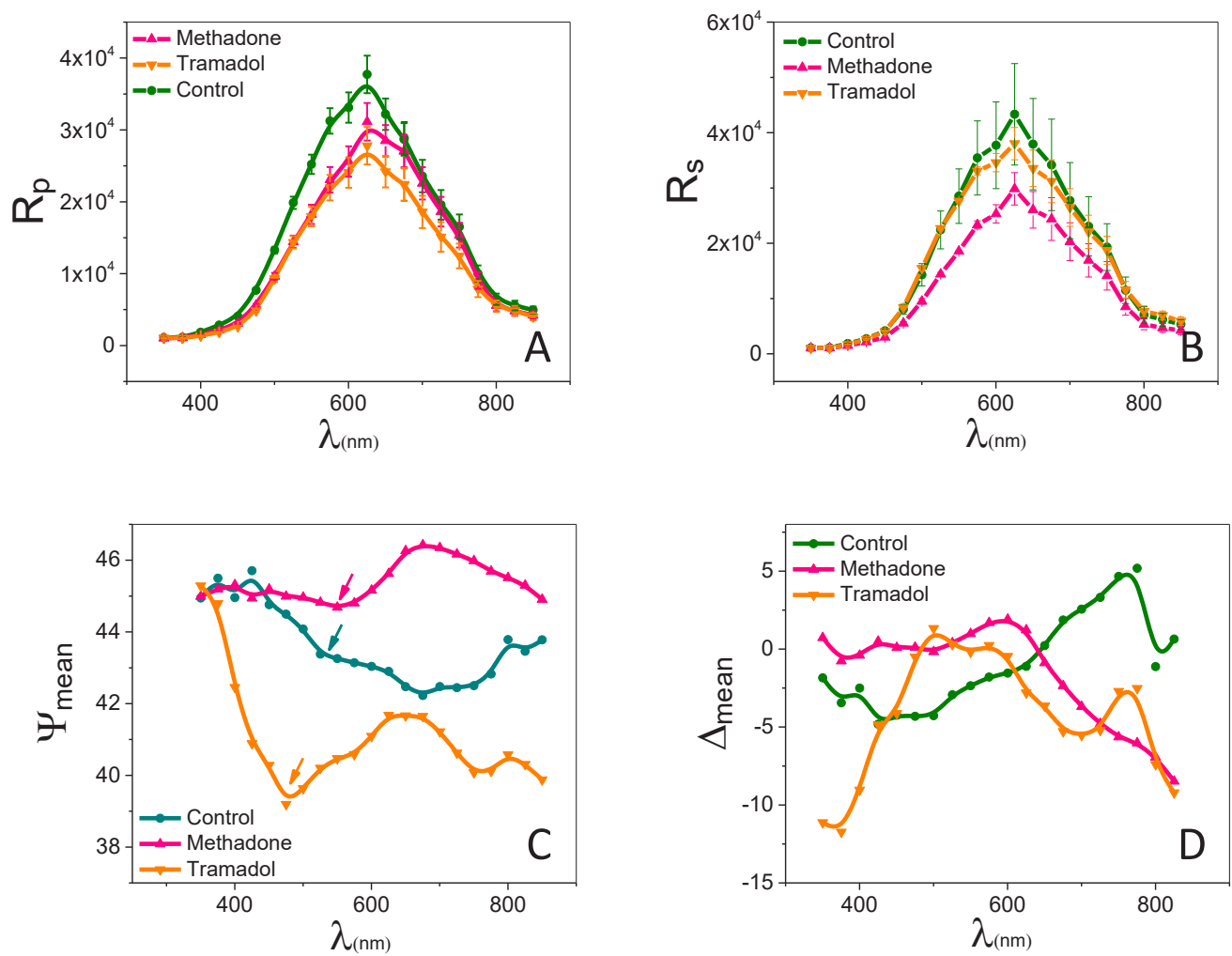

Figure 3. (A, B) The mean values of reflections for $\mathrm{p}$ - and s-polarized incident light. (C, D) Corresponding $\Psi$ and $\Delta$ parameters. The plasmonic resonance dip is not clear in reflection, but this resonance is conspicuous in $\Psi$ and $\Delta$ parameters. The resonance wavelength emerges at $550 \mathrm{~nm}$ for methadone and at $475 \mathrm{~nm}$ for tramadol. The plasmonic resonance is redshifted for the methadone case and blueshifted for the tramadol case in comparison with the control sample.
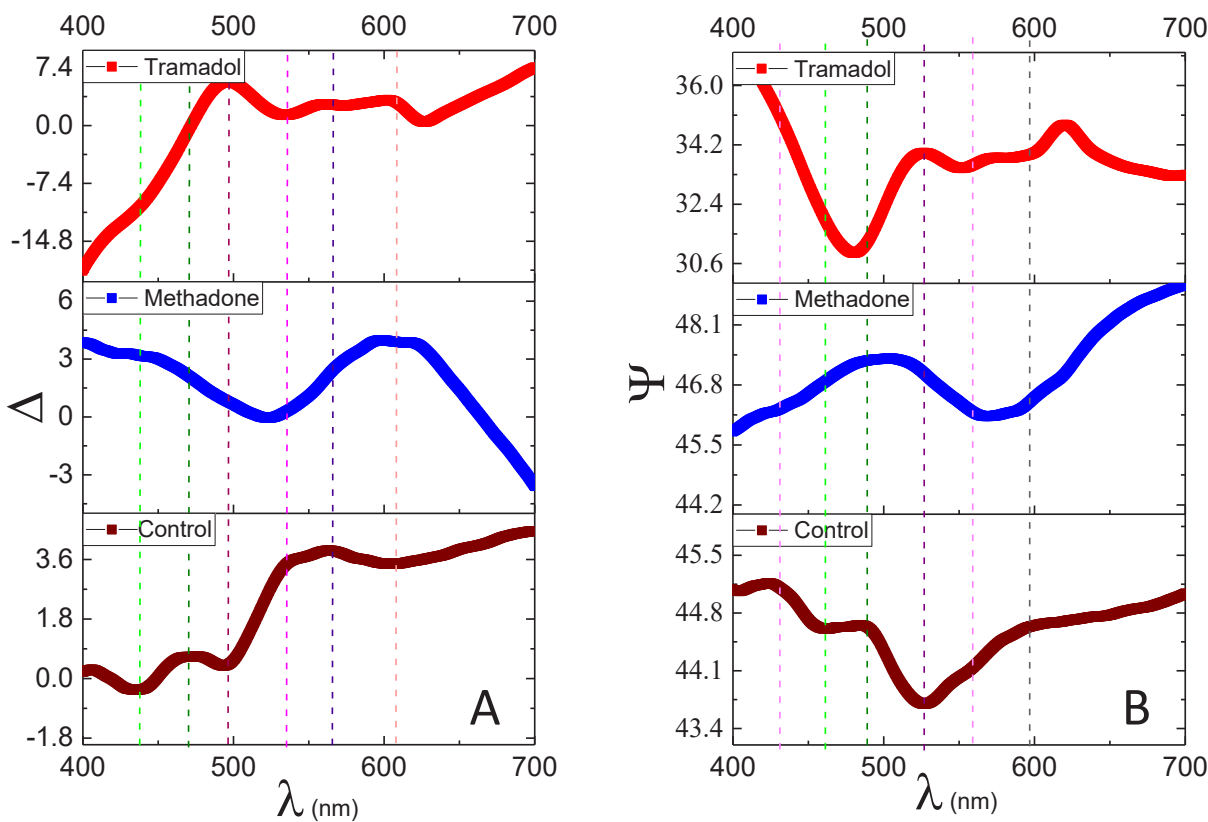

Figure 4. Ellipsometric $\Delta$ (A) and $\Psi$ (B) Parameters for Three Cases: Non-treated Cells (Control), Tramadol-Treated Cells, and MethadoneTreated Cells. Compared to the non-treated case, the spectral position is redshifted for the methadone case and blueshifted for the tramadol case. Drug treatment had a direct effect not only on spectral plasmonic resonance but also on the phase shifts and amplitude ratios. Lightmatter interaction provided bigger phase shifts for the tramadol case in comparison with the methadone case, meaning that the phase change was bigger between $\mathrm{p}$ - and s-polarized light for the tramadol case, but the amplitude ratio underwent more change for methadone. 
case and in comparison, $\Delta_{\text {tramadol }}\left(=5.58^{\circ}\right)>\Delta_{\text {methadone }}$ $\left(=3.88^{\circ}\right)>\Delta_{\text {control }}\left(=3.74^{\circ}\right)$, and the most significant $\Psi$ value was for the methadone case as $\Psi_{\text {methadone }}\left(=46.13^{\circ}\right)>$ $\Psi_{\text {control }}\left(=43.70^{\circ}\right)>\Psi_{\text {tramadol }}\left(=30.92^{\circ}\right)$. It meant that drug treatment had a direct effect on the spectral plasmonic resonance, $\Delta$ and $\Psi$. Additional resonances, which emerged for control and tramadol cases, disappeared for the methadone case. This might be due to the side effects of methadone on $\mu$-opioid receptors in the cell chains. Light-matter interaction provided a bigger phase shift for the tramadol case in comparison with the methadone case, meaning that the phase change was more between $\mathrm{p}$ and s-polarized light for the tramadol case; however, the amplitude ratio underwent more change for methadone. The rate of phase change might directly depend on the time required for the de-polarization of cellular activity under the influence of these two drugs. As shown in Figure 5, we defined a new parameter of $\Delta_{\text {eff }}=\frac{\Delta_{\text {dnng }}-\Delta_{\text {courol }}}{\Delta_{\text {drug }}+\Delta_{\text {conrol }}}$ which showed the contrast between $\Delta_{\text {drug }}$ and $\Delta_{\text {control }}$ in a better way. Correspondingly, we defined $\Psi_{\text {eff }}=\frac{\Psi_{\text {ang }}-\Psi_{\text {connol }}}{\Psi_{\text {dng }}}$ as a new parameter to show the contrast between $\Psi_{\text {drug }}^{\text {andol }}$ and $\Psi_{\text {control }}$. According to the $\Delta_{\text {eff }}$ graph, the tramadol case had the dip and peak values of [ $\left.537 \mathrm{~nm},-0.43^{\circ}\right]$ and [460 $\mathrm{nm}, 1.47^{\circ}$ ], and the methadone case had the dip and peak values of $\left[523 \mathrm{~nm},-1.03^{\circ}\right]$ and $\left[435 \mathrm{~nm}, 1.23^{\circ}\right]$. According to the $\Psi_{\text {eff }}$ graph, the tramadol case had the dip and peak values of $\left[480 \mathrm{~nm},-0.18^{\circ}\right]$ and $\left[526 \mathrm{~nm}, 0.13^{\circ}\right]$, and the methadone case had the dip and peak values of [489 $\mathrm{nm}, 0.03^{\circ}$ ] and [519 nm, 0.04 ${ }^{\circ}$. As seen, we had minor resonances in $\Delta_{\text {eff }}$ and $\Psi_{\text {eff }}$ for the tramadol case. In comparison with the methadone case, tramadol showed a general redshift. Tramadol had bigger absolute $\Delta_{\text {eff }}$ and $\Psi_{\text {eff }}$ values meaning that tramadol caused more contrast in the phase delay and amplitude ratio with the control case in comparison with methadone.

\section{Discussion}

Plasmonic sensing is a strong platform for the detection of the low amount of biomarkers and pharmaceutical drugs. We treated the mesenchymal cells via the sedative drugs of methadone and tramadol and investigated the cellular changes via a highly resolved plasmonic-ellipsometry technique. From a pharmaceutical viewpoint, methadone interacts with more drugs than tramadol (649 vs. 532). ${ }^{39}$ In addition, side disease interaction is higher for methadone compared to tramadol. ${ }^{39}$ In the literature, there are also some comparisons from behavioral/sedative aspects or side effects like anxiety, ${ }^{40}$ cancer pain management, ${ }^{41}$ fatal poisoning ${ }^{42}$ and opiate withdrawal. ${ }^{7}$ We have shown that the drug treatment had a direct effect not only on the spectral plasmonic resonance but also on the phase shifts and amplitude ratios. Light-matter interaction provided a bigger phase shift for the tramadol case compared to the methadone case, meaning that the phase split was larger between $\mathrm{p}$ - and s-polarized light for the tramadol case. However, the amplitude ratio underwent more changes for methadone. This rate of phase change might directly depend on the time required for the de-polarization of cellular activity under the influence of these two drugs. For $\Delta_{\text {eff }}$ and $\Psi_{\text {eff }}$ parameters, tramadol showed a general redshift in comparison with the methadone case. Tramadol had bigger absolute $\Delta_{\text {eff }}$ and $\Psi_{\text {eff }}$ values meaning that tramadol caused more contrast in the phase delay and amplitude ratio with the control case in comparison with methadone. In addition, we showed that the ratio of reflections under $\mathrm{p}$ - and s-polarized incident light $\left(\mathrm{R}_{\mathrm{p}} / \mathrm{R}_{\mathrm{s}}\right)$ for the resonance wavelength in the visible range for simulation and experiment satisfyingly matched. Although there were similarities regarding the effect of tramadol and methadone on the patients besides their similar side effects, we showed that they caused different optical responses on a cell scale.

\section{Conclusion}

In this study, we have shown the effect of methadone and tramadol sedative drugs on stem cell signaling via a plasmonic platform. Tramadol had larger absolute $\Delta_{\text {eff }}$ and $\Psi_{\text {eff }}$ values in comparison with methadone showing that tramadol differentiated more optical responses for p- and s-polarized lights compared to methadone. The proposed technique also has the capability to detect the concentration of the drug, which can play an important role in the clinical detection system in which a safe prescribed dosage of a drug can be tested using optical techniques on a cell scale.
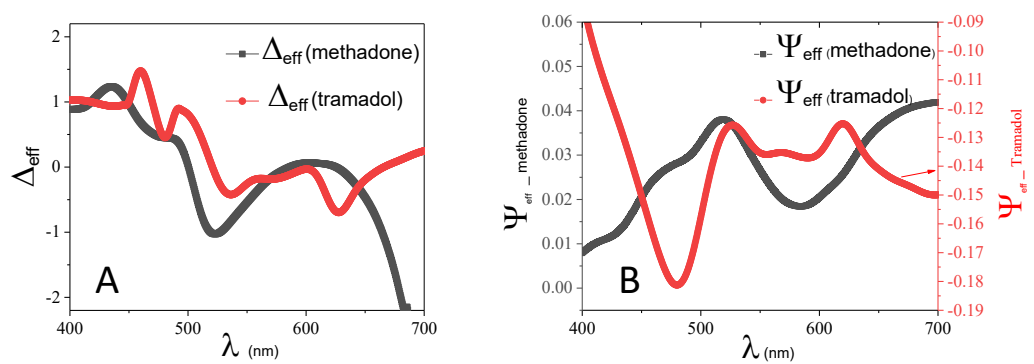

Figure 5. $\Delta_{\text {eff }}$ (A) and $\Psi_{\text {eff }}$ (B) or Cultured Chips for Methadone- and Tramadol-Treated Cells. Tramadol had bigger absolute $\Delta_{\text {eff }}$ and $\Psi_{\text {eff }}$ values meaning that tramadol caused more contrast in the phase delay and amplitude ratio with the control case in comparison with methadone. 


\section{Conflict of Interests}

The authors declare no conflict of interest.

\section{Data Availability}

The data that support the findings of this study are available from the corresponding author upon reasonable request.

\section{Acknowledgment}

This work was supported by Iran National Science Foundation (INSF) under grant number 97006157.

\section{References}

1. Xia M, Tong JH, Ji NN, Duan ML, Tan YH, Xu JG. Tramadol regulates proliferation, migration and invasion via PTEN/ PI3K/AKT signaling in lung adenocarcinoma cells. Eur Rev Med Pharmacol Sci. 2016;20(12):2573-80.

2. Brawanski K, Brockhoff G, Hau P, Vollmann-Zwerenz A, Freyschlag C, Lohmeier A, et al. Efficacy of D,L-methadone in the treatment of glioblastoma in vitro. CNS Oncol. 2018;7(3):CNS18. doi: 10.2217/cns-2018-0006. Epub 2018 Jun 19.

3. Boshra V. Evaluation of osteoporosis risk associated with chronic use of morphine, fentanyl and tramadol in adult female rats. Curr Drug Saf. 2011;6(3):159-63. doi: 10.2174/157488611797579267.

4. Xia M, Tong JH, Zhou ZQ, Duan ML, Xu JG, Zeng HJ, et al. Tramadol inhibits proliferation, migration and invasion via a2-adrenoceptor signaling in breast cancer cells. Eur Rev Med Pharmacol Sci. 2016;20(1):157-65.

5. Ghaderi A, Banafshe HR, Mirhosseini N, Motmaen M, Mehrzad F, Bahmani F, et al. The effects of melatonin supplementation on mental health, metabolic and genetic profiles in patients under methadone maintenance treatment. Addict Biol. 2019;24(4):754-764. doi: 10.1111/ adb. 12650 .

6. Schwarz RK, Bruce RD, Ball SA, Herme M, Altice FL. Comparison of tuberculin skin testing reactivity in opioiddependent patients seeking treatment with methadone versus buprenorphine: policy implications for tuberculosis screening. Am J Drug Alcohol Abuse. 2009;35(6):439-44. doi: 10.3109/00952990903447741.

7. Zarghami M, Masoum B, Shiran MR. Tramadol versus methadone for treatment of opiate withdrawal: a double-blind, randomized, clinical trial. J Addict Dis. 2012;31(2):112-7. doi: 10.1080/10550887.2012.665728.

8. Nicholson AB, Watson GR, Derry S, Wiffen PJ. Methadone for cancer pain. Cochrane Database Syst Rev. 2017;2(2):CD003971. doi: 10.1002/14651858.CD003971. pub4.

9. Ugur M, Derouiche L, Massotte D. Heteromerization Modulates mu Opioid Receptor Functional Properties in vivo. Front Pharmacol. 2018;9:1240. doi: 10.3389/ fphar.2018.01240.

10. Hung CC, Chiou MH, Teng YN, Hsieh YW, Huang CL, Lane HY. Functional impact of ABCB1 variants on interactions between P-glycoprotein and methadone. PLoS One. 2013;8(3):e59419. doi: 10.1371/journal.pone.0059419.

11. Salehi M, Barekateyn M, Amanatkar M. Tramadol versus methadone for the management of acute opioid withdrawal: an add-on study. J Res Med Sci. 2006; 11(3): 185-189.
12. Matsui J, Akamatsu K, Hara N, Miyoshi D, Nawafune H, Tamaki K, Sugimoto N. SPR sensor chip for detection of small molecules using molecularly imprinted polymer with embedded gold nanoparticles. Anal Chem. 2005;77(13):4282-5. doi: 10.1021/ac050227i.

13. Bellassai N, D’Agata R, Jungbluth V, Spoto G. Surface Plasmon Resonance for Biomarker Detection: Advances in Non-invasive Cancer Diagnosis. Front Chem. 2019;7:570. doi: 10.3389/fchem.2019.00570.

14. Myszka DG, Rich RL. Implementing surface plasmon resonance biosensors in drug discovery. Pharm Sci Technol Today. 2000;3(9):310-317. doi: 10.1016/s14615347(00)00288-1.

15. El-Zahry MR. A Localized Surface Plasmon Resonance Sensing Method for Simultaneous Determination of Atenolol and Amiloride in Pharmaceutical Dosage Forms and Urine Samples. J Anal Methods Chem. 2018;2018:9065249. doi: 10.1155/2018/9065249.

16. Izquierdo-Lorenzo I, Alda I, Sanchez-Cortes S, GarciaRamos JV. Adsorption and detection of sport doping drugs on metallic plasmonic nanoparticles of different morphology. Langmuir. 2012;28(24):8891-901. doi: 10.1021/la300194v.

17. Juhász Á, Luty-Błocho M, Wojnicki M, Tóth GK, Csapó E. General method for kinetic and thermodynamic evaluation of a receptor model peptide-drug molecule interaction studied by surface plasmon resonance. Microchemical Journal. 2019;147:311-8. doi: 10.1016/j.microc.2019.03.048

18. Kampranis SC, Gormley NA, Tranter R, Orphanides G, Maxwell A. Probing the binding of coumarins and cyclothialidines to DNA gyrase. Biochemistry. 1999;38(7):1967-76. doi: 10.1021/bi982320p.

19. Su M, Jiang Y, Yu F, Yu T, Du S, Xu Y, Yang L, Liu H. Mirrorlike plasmonic capsules for online microfluidic Raman analysis of drug in human saliva and urine. ACS Appl Bio Mater. 2019;2(9):3828-35. doi: 10.1021/acsabm.9b00425

20. Mozneb M, Mirza AM, Li CZ. Non-Invasive PlasmonicBased Real-Time Characterization of Cardiac Drugs on Cardiomyocytes Functional Behavior. Anal Chem. 2020;92(2):2244-2250. doi: 10.1021/acs.analchem.9b04956.

21. Jackman JA, Linardy E, Yoo D, Seo J, Ng WB, Klemme DJ, et al. Plasmonic Nanohole Sensor for Capturing Single Virus-Like Particles toward Virucidal Drug Evaluation. Small. 2016;12(9):1159-66. doi: 10.1002/smll.201501914.

22. Huang L, Wan J, Wei X, Liu Y, Huang J, Sun X, et al. Plasmonic silver nanoshells for drug and metabolite detection. Nat Commun. 2017;8(1):220. doi: 10.1038/ s41467-017-00220-4.

23. Kong W, Wu D, Hu N, Li N, Dai C, Chen X, Set al. Robust hybrid enzyme nanoreactor mediated plasmonic sensing strategy for ultrasensitive screening of anti-diabetic drug. Biosens Bioelectron. 2018;99:653-659. doi: 10.1016/j. bios.2017.08.009.

24. Sohrabi F, Etezadi D, Perin R, Jahani Y, Mohammadi E, Hamidi SM. Phase-sensitive optical neural recording of cerebellum tissue on a flexible interface. J Appl Phys. 2020;127(11):113101. doi: 10.1063/1.5132422

25. Sohrabi F, Hamidi SM. Fabrication methods of plasmonic and magnetoplasmonic crystals: a review. Eur Phys J Plus. 2017;132(1):1-21. doi: 10.1140/epjp/i2017-11294-2 
26. Soleimani M, Nadri S. A protocol for isolation and culture of mesenchymal stem cells from mouse bone marrow. Nat Protoc. 2009;4(1):102-6. doi: 10.1038/nprot.2008.221.

27. Moghadam M, Tokhanbigli S, Baghaei K, Farivar S, Asadzadeh Aghdaei H, Zali MR. Gene expression profile of immunoregulatory cytokines secreted from bone marrow and adipose derived human mesenchymal stem cells in early and late passages. Mol Biol Rep. 2020;47(3):17231732. doi: 10.1007/s11033-020-05264-2.

28. Farivar S, Mohamadzade Z, Shiari R, Fahimzad A. Neural differentiation of human umbilical cord mesenchymal stem cells by cerebrospinal fluid. Iran J Child Neurol. 2015;9(1):87-93.

29. Plantz MA, Hsu WK. Single-level Posterolateral Fusion (PLF) Alone and Posterior Interbody Fusion (PLIF/ TLIF) Alone Lead to a Decreased Risk of Short-term Complications Compared to Combined PLF With PLIF/ TLIF Procedures: A Matched Analysis. Spine (Phila Pa 1976). 2020;45(21):E1391-E1399. doi: 10.1097/ BRS.0000000000003615.

30. Saeidifard S, Sohrabi F, Ghazimoradi MH, Hamidi SM, Farivar S, Ansari MA. Two-dimensional plasmonic biosensing platform: Cellular activity detection under laser stimulation. J Appl Phys. 2019;126(10):104701. doi: $10.1063 / 1.5111533$

31. Sohrabi F, Jahani Y, Sanchez-Mut JV, Mohammadi E, Barzegar Z, Li X, et al. Membrane activity detection in cultured cells using phase-sensitive plasmonics. Opt Express. 2020;28(24):36643-36655. doi: 10.1364/ OE.399713.

32. Sohrabi F, Hamidi SM. Neuroplasmonics: From Kretschmann configuration to plasmonic crystals. Eur Phys J Plus. 2016;131(7):1-5. doi: 10.1140/epjp/i2016-16221-5

33. Sohrabi F, Hamidi SM, Asgari N, Ansari MA, Gachiloo R. One dimensional photonic crystal as an efficient tool for in-vivo optical sensing of neural activity. Opt Mat. 2019;96:109275. doi: 10.1016/j.optmat.2019.109275
34. Oates TW, Wormeester H, Arwin H. Characterization of plasmonic effects in thin films and metamaterials using spectroscopic ellipsometry. Prog Surf Sci. 2011;86(1112):328-76. doi: 10.1016/j.progsurf.2011.08.004

35. Sohrabi F, Hamidi SM. Optical detection of brain activity using plasmonic ellipsometry technique. Sensor Actuat B-Chem. 2017;251:153-63. doi: 10.1016/j.snb.2017.05.037.

36. Sohrabi F, Etezadi D, Perin R, Jahani Y, Mohammadi E, Hamidi SM. Phase-sensitive optical neural recording of cerebellum tissue on a flexible interface. J Appl Phys. 2020;127(11):113101. doi: 10.1063/1.5132422

37. Haddawi SF, Mirahmadi M, Mbarak H, Kodeary AK, Ghasemi M, Hamidi SM. Footprint of plexcitonic states in low-power green-blue plasmonic random laser. Appl Phys A. 2019;125(12):1-9. doi: 10.1007/s00339-019-3139-y

38. Mbarak H, Ghahrizjani RT, Hamidi SM, Mohajerani E, Zaatar Y. Reversible and tunable photochemical switch based on plasmonic structure. Sci Rep. 2020;10(1):5110. doi: 10.1038/s41598-020-62058-z.

39. American Society of Health-System Pharmacists, Cerner Multum and IBM Watson Micromedex. Comparing Methadone vs Tramadol. Accessed January 11, 2020. https://www.drugs.com/compare/methadone-vs-tramadol

40. Gholami M, Saboory E, Ahmadi AA, Asouri M, Nasirikenari M, Rostamnezhad M. Long-time effects of prenatal morphine, tramadol, methadone, and buprenorphine exposure on seizure and anxiety in immature rats. Int J Neurosci. 2020;130(9):898-905. doi: 10.1080/00207454.2019.1709841.

41. Portenoy RK, Mehta Z, Ahmed E. Cancer pain management with opioids: optimizing analgesia. In : UpToDate, Waltham, MA (Accessed on January 04, 2020.)

42. Häkkinen $M$, Launiainen $T$, Vuori E, Ojanperä I. Comparison of fatal poisonings by prescription opioids. Forensic Sci Int. 2012;222(1-3):327-31. doi: 10.1016/j. forsciint.2012.07.011. 